\title{
Câncer na infância: análise comparativa da incidência, mortalidade e sobrevida em Goiânia (Brasil) e outros países
}

\author{
Childhood cancer: a comparative analysis \\ of incidence, mortality, and survival in Goiania \\ (Brazil) and other countries
}

Patrícia Emília Braga 1

Maria do Rosário Dias de Oliveira Latorre 1 Maria Paula Curado 2

\footnotetext{
1 Departamento de Epidemiologia, Faculdade de Saúde Pública, Universidade de São Paulo. Av. Dr. Arnaldo 715, São Paulo, SP 01246-904, Brasil. bragapat@netpoint.com.br mdrddola@usp.br 2 Registro de Câncer, Associação de Combate ao Câncer em Goiás. Rua 239 no 206, Setor Universitário, Goiânia, GO 74605-070, Brasil.
}

\begin{abstract}
Analysis of cancer incidence, mortality, and survival rates can yield geographic and temporal trends that are useful for planning and evaluating health interventions. This article reviews cancer incidence and mortality rates and respective trends around the world in children under 15 years old, as well as their 5-year survival rates in developed and developing countries. We conclude that even though increasing or stable childhood cancer incidence rates and decreasing mortality rates have been observed in developed countries, the trends remain unknown in developing countries. Data from the city of Goiania, Brazil, show stable childhood cancer incidence and mortality rates. Five-year survival rates (48\%) in Goiania are similar to those seen in underdeveloped regions and lower than those reported in developed countries (64-70\%).

Key words Infant Mortality; Neoplasm; Survival Rate
\end{abstract}

Resumo As análises de incidência do câncer, mortalidade e sobrevida permitem identificar variações geográficas e temporais importantes para o planejamento e avaliação de ações de saúde. Neste trabalho, apresentam-se os coeficientes de incidência e de mortalidade por câncer em menores de 15 anos e suas tendências em vários países do mundo, assim como as probabilidades acumuladas de sobrevida após cinco anos do diagnóstico, em regióes desenvolvidas ou em desenvolvimento. Conclui-se que, embora em países desenvolvidos observem-se taxas crescentes ou estáveis de incidência e decrescentes de mortalidade para o câncer infantil, suas tendências são desconhecidas nos países em desenvolvimento. Nos dados de Goiânia analisados, observaram-se taxas estáveis de incidência e de mortalidade para o câncer infantil, e a sobrevida após cinco anos de diagnóstico (48\%) mostrou-se semelhante à de outras regiões em desenvolvimento e inferior às observadas em regiões desenvolvidas (64\%-70\%).

Palavras-chave Mortalidade Infantil; Neoplasias; Taxa de Sobrevivência 
A análise descritiva-quantitativa em estudos epidemiológicos utiliza parâmetros como os coeficientes de mortalidade e de incidência para, a partir desses, detectar eventuais variações geográficas e temporais, que permitam gerar hipóteses sobre possíveis fatores de risco segundo os diversos tipos de câncer. Os resultados de tais análises representam instrumentos fundamentais para o planejamento e acompanhamento das ações de saúde, visando a uma melhor abordagem assistencial e preventiva.

Dados relativos à incidência de câncer podem ser obtidos consultando-se os Registros de Câncer de Base Populacional, que vêm sendo implantados nas últimas décadas (Coleman et al., 1993). Atualmente, existem mais de duzentos registros como esses em todo o mundo, sendo 18 no Brasil. No entanto, $67 \%$ dos registros brasileiros iniciaram suas atividades há menos de três anos. Estes coletam dados sobre todos os casos novos de câncer diagnosticados em uma área geográfica delimitada, permitindo detectar áreas onde a população é mais afetada pela doença e auxiliando no planejamento e estabelecimento de programas de prevenção e tratamento (Jensen \& Storm, 1991; Latorre \& Franco, 1996).

Em muitos países onde inexistem dados sobre a incidência de câncer, os estudos epidemiológicos valem-se das taxas de mortalidade como indicadoras do impacto da doença na população e preditoras das taxas de morbidade (Franco, 1997; Latorre, 1992). Deve-se, no entanto, lembrar que os tumores associados a um melhor prognóstico serão sempre subestimados nesta abordagem.

\section{Câncer em menores de 15 anos}

Em geral, as neoplasias mais freqüentes em adultos dificilmente ocorrem em crianças. Além disso, os tumores infantis, entendidos como o conjunto de neoplasias que acometem os menores de 15 anos, diferem dos tumores típicos de adultos em relação à sua localização, tipo histológico e comportamento clínico (Little, 1999; Malkin, 1997). Os tumores infantis correspondem a um grupo altamente específico, geralmente embrionário, do sistema reticuloendotelial, do sistema nervoso central, do tecido conectivo e de vísceras, ao passo que tumores epiteliais são extremamente raros nessa faixa etária (Fundação Antônio Prudente, 2001). Assim, entre menores de 15 anos de idade, diagnosticam-se mais freqüentemente leucemias, linfomas, tumores do sistema nervoso central e do sistema simpático, rabdomiossar- comas, tumor de Wilms, retinoblastomas e tumores ósseos (Bleyer, 1990; Breslow \& Langholz, 1983; Franco, 1997; Parkin et al., 1988a; Ries et al., 1999a), enquanto, entre adultos, predominam os tumores pulmonares, do estômago, de mama, do intestino e do fígado (Parkin et al., 2001).

As faixas etárias pediátricas mais precoces (0 a 4 anos) são as mais propensas ao desenvolvimento de câncer (Petrilli et al., 1997), com exceção de linfomas, carcinomas e tumores ósseos, que predominam em crianças entre $10 \mathrm{e}$ 14 anos. Sabe-se ainda que, do ponto de vista clínico-evolutivo, os tumores infantis tendem a apresentar menores períodos de latência, quase sempre crescem rapidamente, são agressivamente invasivos e respondem melhor à quimioterapia (Little, 1999).

\section{Incidência}

O câncer infantil compreende de $0,5 \%$ a $3 \%$ de todas as neoplasias na maioria das populações (Parkin et al., 1988b), estimando-se uma incidência anual de cerca de duzentos mil casos em todo o mundo (Bleyer, 1990).

Recentemente Parkin et al. (1998) publicaram dados sobre a incidência do câncer infantil em aproximadamente sessenta países, incluindo as nações desenvolvidas ou em desenvolvimento. A esses dados podem-se acrescentar resultados obtidos por Braga (2000) no Município de Goiânia (Goiás, Brasil). A Tabela 1 resume os resultados alcançados nas publicações citadas, para algumas áreas geográficas.

Verifica-se que, geralmente, as taxas de incidência para o sexo feminino são menores do que as encontradas para o sexo masculino. No entanto, em Goiânia, a razão dos coeficientes de incidência entre os sexos (masculino/feminino) foi inferior a um. Entre os meninos, um dos mais altos coeficientes padronizados de incidência anual de câncer infantil foi registrado em Kampala, Uganda (225,6 por um milhão de meninos, no período de 1992 a 1995); já entre as meninas, as maiores taxas referem-se à Croácia (154,8; de 1987 a 1990). A Namíbia apresentou, entre 1983 e 1992, os menores coeficientes observados em ambos os sexos (49,6 por um milhão de meninos e 42,2 para as meninas). Wessels et al. (1998), no entanto, crêem que os baixos coeficientes relatados na Namíbia possam refletir subnotificação de casos nesse país ou mesmo a dificuldade de diagnosticar corretamente as neoplasias infantis, seja pelo fato de as crianças não serem levadas aos serviços de saúde, seja por falecerem prematuramente em virtude de enfermidades infecciosas. Wa- 
binga et al. (1998) atribuem as altas taxas observadas em Uganda às altas incidências do sarcoma de Kaposi (84,4 e 52,2 por um milhão de meninos e meninas, respectivamente) e do linfoma de Burkitt (48,5 e 25,2 por um milhão de meninos e meninas, respectivamente) encontradas na região.

Ao analisar-se a incidência estratificada segundo os grupos diagnósticos das neoplasias infantis, verificam-se os resultados ilustrados na Tabela 2.

Dentre todas as neoplasias infantis, as leucemias representam as mais freqüentemente diagnosticadas, sendo responsáveis, na maioria das populações, por $25 \%$ a $35 \%$ de todas as neoplasias malignas pediátricas. Porém, na África, as leucemias são predominantes apenas na Argélia (37\%) e no Zimbábue (21\%), compreendendo, nos demais países, apenas de $10 \%$ a 15\% dos tumores infantis. Prevalências superiores a $40 \%$ foram encontradas na Costa Rica (42\%), no Equador (45\%), em Hong Kong (41\%) e nas Filipinas (48\%) (Parkin et al., 1998). Em Goiânia, entre 1989 e 1996, foram as neoplasias mais comuns, concentrando $27 \%$ de todos os tumores infantis $(22,8 \%$ para as meninas e $31,5 \%$ para os meninos) (Braga, 2000).

Na maioria dos países, crianças menores de cinco anos são as mais freqüentemente acometidas por este tipo de neoplasia.

Dentre as leucemias, as mais comuns são as agudas e, dentre estas, a leucemia linfocítica aguda (LLA), totalizando, nas populações brancas da América do Norte, Oceania e Europa, de $75 \%$ a $80 \%$ de todas as leucemias. Nas mesmas regiões, a leucemia não linfocítica aguda (LNLA) compreende de $15 \%$ a $17 \%$ dos casos. Nada comum na faixa etária infantil, a leucemia mielóide crônica (LMC) raramente excede a proporção de 4\% (Sharp et al., 1999). No Município de Goiânia, no período de 1989 a 1996, essas freqüências foram de $66 \%$ para as LLA, $20 \%$ para as LNLA e 1,4\% para as LMC (Braga, 2000).

Os tumores do sistema nervoso central (SNC) representam, principalmente nos países desenvolvidos, o segundo grupo de diagnóstico mais comum na infância, contribuindo com cerca de $19 \%$ a $27 \%$ das neoplasias. Dados análogos foram observados em Goiânia, com prevalência de 18,3\% (Braga, 2000). Na maioria dos países africanos, ao contrário, sua freqüência é inferior a 5\% (Parkin et al., 1998). Nesta topografia, em particular, a classificação adotada pela Agência Internacional de Pesquisa em Câncer (IARC) inclui neoplasias benignas e malignas. Os tipos histológicos mais comuns em menores de 15 anos correspondem aos astrocitomas e meduloblastomas.

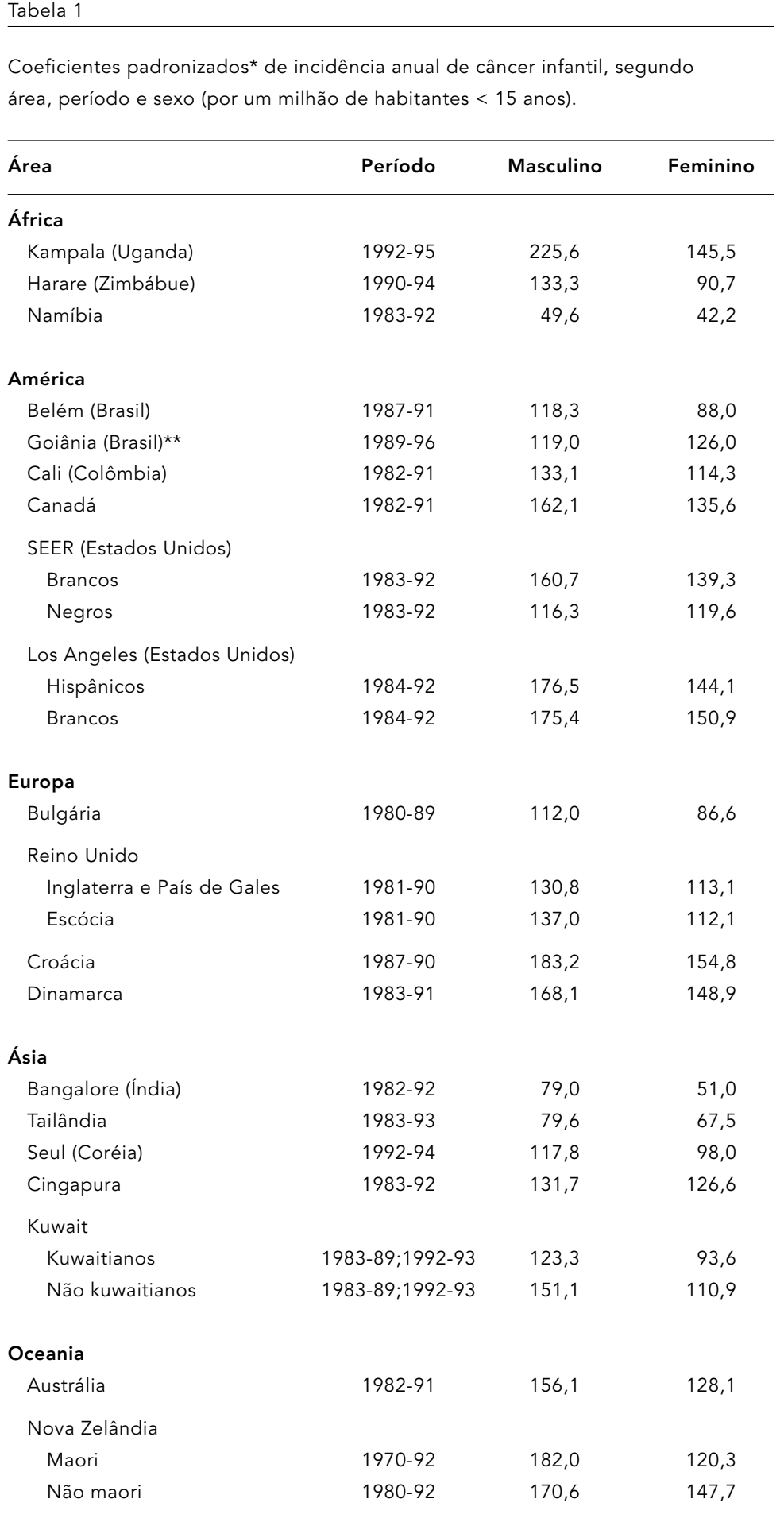

* população padrão: mundial de Segi de 1960

Fontes: Parkin et al., 1998; ** Braga, 2000

SEER = Surveillance, Epidemiology and End Results 
Tabela 2

Coeficientes padronizados* de incidência anual de câncer infantil, segundo grupo de diagnóstico

e sexo (por um milhão de habitantes < 15 anos).

\begin{tabular}{|c|c|c|c|c|}
\hline \multirow[t]{2}{*}{ Grupo de diagnóstico } & \multicolumn{2}{|c|}{ Feminino } & \multicolumn{2}{|c|}{ Masculino } \\
\hline & menor & maior & menor & maior \\
\hline Leucemias & $\begin{array}{l}4,2 \\
\text { Mali }\end{array}$ & $\begin{array}{l}54,3 \\
\text { Estados Unidos } \\
\text { (Los Angeles) } \\
\text { (hispânicos) }\end{array}$ & $\begin{array}{l}2,1 \\
\text { Mali }\end{array}$ & $\begin{array}{l}64,5 \\
\text { Hong Kong }\end{array}$ \\
\hline Linfomas & $\begin{array}{l}3,4 \\
\text { Namíbia }\end{array}$ & $\begin{array}{l}39,9 \\
\text { Uganda }\end{array}$ & $\begin{array}{l}8,2 \\
\text { Namíbia }\end{array}$ & $\begin{array}{l}67,5 \\
\text { Uganda }\end{array}$ \\
\hline $\begin{array}{l}\text { Tumores do sistema } \\
\text { nervoso central }\end{array}$ & $\begin{array}{l}2,1 \\
\text { Mali }\end{array}$ & $\begin{array}{l}43,2 \\
\text { Suécia }\end{array}$ & $\begin{array}{l}0,7 \\
\text { Mali }\end{array}$ & $\begin{array}{l}43,2 \\
\text { Croácia }\end{array}$ \\
\hline $\begin{array}{l}\text { Tumores do sistema } \\
\text { nervoso simpático }\end{array}$ & $\begin{array}{l}0,4 \\
\text { Nigéria }\end{array}$ & $\begin{array}{l}14,5 \\
\text { Israel (judeus) }\end{array}$ & $\begin{array}{l}1,0 \\
\text { Brasil (Goiânia) }\end{array}$ & $\begin{array}{l}17,2 \\
\text { Nova Zelândia } \\
\text { (maori) }\end{array}$ \\
\hline Retinoblastoma & $\begin{array}{l}0,7 \\
\text { Bulgária } \\
\text { e Kuwait } \\
\text { (kuwaitianos) }\end{array}$ & $\begin{array}{l}19,5 \\
\text { Mali }\end{array}$ & $\begin{array}{l}0,4 \\
\text { Argélia }\end{array}$ & $\begin{array}{l}29,4 \\
\text { Mali }\end{array}$ \\
\hline Tumores renais & $\begin{array}{l}1,3 \\
\text { Kuwait } \\
\text { (kuwaitianos) }\end{array}$ & $\begin{array}{l}17,7 \\
\text { Croácia }\end{array}$ & $\begin{array}{l}1,7 \\
\text { Vietnã (Hanói) }\end{array}$ & $\begin{array}{l}17,4 \\
\text { Zimbábue }\end{array}$ \\
\hline Tumores hepáticos & $\begin{array}{l}0,2 \\
\text { Porto Rico }\end{array}$ & $\begin{array}{l}3,5 \\
\text { Mali }\end{array}$ & $\begin{array}{l}0,4 \\
\text { Namíbia }\end{array}$ & $\begin{array}{l}4,8 \\
\text { Vietnã (Hanói) }\end{array}$ \\
\hline $\begin{array}{l}\text { Tumores malignos } \\
\text { dos ossos }\end{array}$ & $\begin{array}{l}0,8 \\
\text { Estados Unidos } \\
\text { (Los Angeles) } \\
\text { (negros) }\end{array}$ & $\begin{array}{l}11,7 \\
\text { Brasil (Goiânia) }\end{array}$ & $\begin{array}{l}2,2 \\
\text { Cingapura }\end{array}$ & $\begin{array}{l}9,8 \\
\text { Nova Zelândia }\end{array}$ \\
\hline $\begin{array}{l}\text { Sarcomas das } \\
\text { partes moles }\end{array}$ & $\begin{array}{l}1,6 \\
\text { Argélia }\end{array}$ & $\begin{array}{l}60,0 \\
\text { Uganda }\end{array}$ & $\begin{array}{l}1,5 \\
\text { Argélia }\end{array}$ & $\begin{array}{l}88,2 \\
\text { Uganda }\end{array}$ \\
\hline $\begin{array}{l}\text { Neoplasias gonadais e } \\
\text { das células germinativas }\end{array}$ & $\begin{array}{l}0,4 \\
\text { Argélia }\end{array}$ & $\begin{array}{l}9,9 \\
\text { Japão (Osaka) }\end{array}$ & $\begin{array}{l}0,4 \\
\text { Argélia }\end{array}$ & $\begin{array}{l}9,7 \\
\text { Nova Zelândia } \\
\text { (maori) }\end{array}$ \\
\hline $\begin{array}{l}\text { Carcinomas e neoplasias } \\
\text { epiteliais }\end{array}$ & $\begin{array}{l}0,7 \\
\text { Brasil (Belém) } \\
\text { e Índia (Delhi) }\end{array}$ & $\begin{array}{l}8,3 \\
\text { Suécia }\end{array}$ & $\begin{array}{l}0,8 \\
\text { Alemanha } \\
\text { (República } \\
\text { Federal) }\end{array}$ & $\begin{array}{l}10,1 \\
\text { Uganda }\end{array}$ \\
\hline
\end{tabular}

* população padrão: mundial de Segi de 1960

Fonte: Parkin et al. (1998).

Os linfomas, seguindo-se aos tumores do sistema nervoso central, constituem o terceiro tipo de neoplasia de maior incidência nos países desenvolvidos, abrangendo de $7 \%$ a $18 \%$ dos casos de neoplasia infantil. Nos países em desenvolvimento, geralmente, ocupam a segunda posição nas taxas de incidência, situação essa confirmada pelo estudo recentemente conduzido em Goiânia, no qual totalizavam $18,3 \%$ dos tumores infantis diagnosticados en- tre 1989 e 1996 (Braga, 2000). Observa-se, no entanto, grande variabilidade dos linfomas em termos histológicos quando regiões diferentes são comparadas. Aproximadamente $45 \%$ do total dos linfomas em crianças são representados pelo linfoma de Hodgkin (Greenberg \& Shuster, 1985) e sua incidência é, comumente, mais acentuada em populações com pior nível sócio-econômico, como as do Kuwait, Brasil e Costa Rica (Parkin et al., 1998). Em Goiânia, por 
exemplo, $44 \%$ dos linfomas acima mencionados eram de Hodgkin. Outro tipo comum de linfoma é o de Burkitt, endêmico nos países africanos situados ao sul do Saara, onde, na década de 70, chegou a ser responsável por mais de $90 \%$ de todos os casos de linfomas, representando o tumor infantil mais freqüentemente diagnosticado (Sharp et al., 1999). Em Kampala (Uganda), no período de 1992 a 1995, verificou-se um coeficiente de incidência dessa neoplasia de 36,1 para cada um milhão de menores de 15 anos. O linfoma de Burkitt é comum também em Papua Nova Guiné, onde o vírus de Epstein-Barr é considerado endêmico; entre 1979 e 1988 esse tumor foi responsável por $58 \%$ dos casos de linfomas diagnosticados naquele país (Parkin et al., 1998).

Na Tabela 3, resumem-se as tendências de incidência do câncer infantil em diferentes regiões do mundo.

Observa-se que, nos Estados Unidos, entre as décadas de 70 e 80 , houve um aumento médio anual de $1 \%$ nos índices de incidência de todas as neoplasias malignas combinadas (Bunin et al., 1996; Gurney et al., 1996), de 1,8\% para as leucemias linfocíticas agudas e, também, para os tumores do SNC. Para estes últimos, esse acréscimo foi mais acentuado entre 1983 e 1986 (Linet et al., 1999; Smith et al., 1998). Particularizando as crianças com idade igual ou inferior a 12 meses, essas tendências mostram-se mais evidentes, com aumento médio anual de $2,9 \%$ na incidência de todas as neoplasias e de $4,1 \%$ para os tumores do SNC, no período de 1973 a 1992 (Gurney et al., 1997). Contudo, na maioria das neoplasias pediátricas não houve aumentos ou declínios com significância estatística.

De acordo com Gibbons et al. (1994), desde 1969 a incidência de câncer em crianças tem aumentado gradualmente no Canadá, porém com variações significativas entre suas províncias: houve aumento de incidência na província de Quebec, enquanto decréscimo da doença pôde ser verificado por Coleman et al. (1993) nas províncias marítimas (Nova Scotia, New Brunswick, Prince Edward Island). Em Porto Rico e em Cali (Colômbia), o aumento nas taxas de incidência também foi significativo.

Na Europa, verifica-se expressiva variação entre os coeficientes de incidência de câncer na infância (Coleman et al., 1993; Levi et al., 1992). As cidades de Zaragoza e Navarra, na Espanha, apresentaram aumento estatisticamente significativo nas taxas de incidência de todas as neoplasias malignas em crianças de 0 a 14 anos de idade (Pollán et al., 1997), que se deu principalmente em virtude do crescimento ob- servado nos coeficientes de incidência dos tumores do SNC.

Na Dinamarca, entre 1943 e 1984, apesar de incrementos com significância estatística terem sido observados na incidência dos tumores do SNC - linfomas e neuroblastomas no sexo masculino -, não foi observada tendência de aumento para todas as neoplasias infantis combinadas (De Nully-Brown et al., 1989). A incidência de leucemias manteve-se constante no período de 1980 a 1992 na Alemanha (Kaatsch et al., 1995), enquanto os tumores do SNC apresentaram aumento em suas taxas até 1987 , tendo-se estabilizado após este período.

Na Austrália, McWhirter et al. (1996) observaram aumento estatisticamente significativo nas taxas de incidência para todas as neoplasias malignas em todas as crianças de 0 a 14 anos, às custas do grupo etário de 10 a 14 e ainda para o conjunto de meninas. Todavia, particularmente no Estado de Victoria, notou-se estabilidade na incidência de todas as neoplasias combinadas e de todos os subgrupos individualizados (Giles et al., 1995).

Os aumentos verificados na incidência de todas as neoplasias malignas infantis são muitas vezes justificados como, principalmente, decorrentes de incrementos na incidência de leucemias e de tumores do SNC. Contudo, tais acréscimos podem não significar de fato que a ocorrência da doença tenha realmente se alterado na população. Assim, Miller et al. (1995) atribuíram o aumento na incidência das LLA em crianças dos Estados Unidos, entre 1973 e 1987, ao aprimoramento nas técnicas diagnósticas e à maior especificidade das mesmas, fazendo com que leucemias classificadas anteriormente apenas como agudas, passassem a ser melhor caracterizadas como LLA.

Da mesma forma, os aumentos significativos observados nos coeficientes de incidência dos tumores do SNC, encontrados em países desenvolvidos, como Espanha, Dinamarca, Estados Unidos e Alemanha, são justificados como resultantes do recente aprimoramento nas técnicas de diagnóstico, advindo da incorporação de métodos mais sofisticados de diagnóstico por imagem, que incluem a tomografia computadorizada (De Nully-Brown et al., 1989; Pollán et al., 1997) e, mais recentemente, a ressonância nuclear magnética, no caso dos tumores do SNC (Black, 1998; Smith et al., 1998). Com essa suposição, Desmeules et al. (1992), revendo casuística da América do Norte, notaram que $20 \%$ dos tumores cerebrais não teriam sido diagnosticados na ausência das técnicas de neuroimagem. Além disso, observou-se, nos Estados Unidos, que o aumento na incidência 
Tabela 3

Tendências da incidência do câncer infantil no mundo e da mortalidade por esta doença.

\begin{tabular}{|c|c|c|c|}
\hline Local & Autores & Período & Tendências \\
\hline \multicolumn{4}{|l|}{ Incidência } \\
\hline \multicolumn{4}{|l|}{ América } \\
\hline Estados Unidos & Linet et al. (1999) & $1975-95$ & $\begin{array}{l}\text { Estável para as leucemias; aumento na } \\
\text { incidência dos tumores do SNC; declínio } \\
\text { na incidência dos linfomas }\end{array}$ \\
\hline Brasil (Goiânia) & Braga (2000) & $1989-96$ & Estável para ambos os sexos \\
\hline \multicolumn{4}{|l|}{ Europa } \\
\hline Dinamarca & $\begin{array}{l}\text { De Nully-Brown } \\
\text { et al. (1989) }\end{array}$ & $1943-84$ & Estável para ambos os sexos \\
\hline Província de Torino (Itália) & Mosso et al. (1992) & $1967-86$ & Estável para ambos os sexos \\
\hline Zaragoza e Navarra (Espanha) & Pollán et al. (1997) & $1973-87$ & $\begin{array}{l}\text { Aumento na incidência de todas } \\
\text { as neoplasias malignas em crianças }\end{array}$ \\
\hline \multicolumn{4}{|l|}{ Oceania } \\
\hline Austrália & McWhirter et al. (1996) & $1982-91$ & $\begin{array}{l}\text { Estável para o sexo masculino, porém } \\
\text { crescente para o feminino }\end{array}$ \\
\hline \multicolumn{4}{|l|}{ Mortalidade } \\
\hline \multicolumn{4}{|l|}{ América } \\
\hline Estados Unidos & Linet et al. (1999) & $1975-95$ & $\begin{array}{l}\text { Declínio de } 58 \% \text { na mortalidade para } \\
\text { todas as neoplasias malignas em crianças }\end{array}$ \\
\hline Canadá & Levi et al. (1995) & $1950-89$ & Decrescente para ambos os sexos \\
\hline Brasil (Goiânia) & Braga (2000) & $1989-96$ & Decrescente para os maiores de 4 anos \\
\hline \multicolumn{4}{|l|}{ Europa } \\
\hline ex-União Soviética & La Vecchia et al. (1994) & $1965-90$ & Estável para ambos os sexos \\
\hline Província de Torino (Itália) & Mosso et al. (1992) & $1967-86$ & $\begin{array}{l}\text { Declínio na mortalidade para todas } \\
\text { as neoplasias malignas em crianças }\end{array}$ \\
\hline Israel & Levi et al. (1995) & $1970-89$ & Decrescente para ambos os sexos \\
\hline Áustria & Kunze et al. (1997) & $1980-92$ & $\begin{array}{l}\text { Declínio na mortalidade para todas } \\
\text { as neoplasias malignas em crianças }\end{array}$ \\
\hline \multicolumn{4}{|l|}{ Oceania } \\
\hline Austrália & Levi et al. (1995) & $1950-89$ & Decrescente para ambos os sexos \\
\hline
\end{tabular}

$\mathrm{SNC}=$ sistema nervoso central.

de tumores malignos primários do sistema nervoso central em crianças deu-se de forma abrupta em meados da década de 80, o que sugere a influência de novas abordagens diagnósticas na identificação desses tumores (Black, 1998; Smith et al., 1998).

Já no estudo realizado em Goiânia, entre 1989 e 1996, observou-se declínio, com significância estatística, nas taxas de incidência de todos os tumores em crianças de 0 a 4 anos e dentre aquelas com idade entre 10 e 14 anos (Braga, 2000). Entretanto, os coeficientes se mantiveram estáveis na análise conjunta para todas as faixas etárias.

\section{Mortalidade}

No que tange à mortalidade, o câncer tem-se destacado como uma importante causa entre os menores de 15 anos. Segundo Little (1999), as neoplasias infantis representam hoje um problema de saúde pública ainda maior do que no passado, devido ao maior controle das doenças transmissíveis.

Nos países desenvolvidos, o câncer representa a segunda causa de morte mais freqüente em crianças, seguindo-se aos acidentes (Greenlee et al., 2001; Little, 1999). Nos Estados Unidos da América, verificam-se aproximadamente 1.300 óbitos por câncer infantil ao ano, 
sendo a doença responsável por $10 \%$ das mortes nessa faixa etária (Bleyer, 1990; Greenlee et al., 2001).

Entre os países da comunidade européia, no período de 1979 a 1988, o câncer determinou, na faixa etária pediátrica, aproximadamente 3.400 óbitos ao ano (Martos \& Olsen, 1993). No entanto, observa-se que existe grande variabilidade dos coeficientes de mortalidade por câncer infantil em diferentes regiões: na Europa, os países do sudeste apresentam coeficientes $50 \%$ a $100 \%$ maiores do que os observados na maioria dos países do norte, entre 1950 e 1989 (Levi et al., 1992).

No Brasil, as estatísticas sobre as neoplasias infantis têm sido pouco analisadas na literatura médica nacional, apesar da existência de fontes de dados, tais como o Sistema de Informação de Mortalidade do Ministério da Saúde (DATASUS, 1998) e os Registros de Câncer de Base Populacional em funcionamento no País. Sabe-se que, no ano de 1994, entre as crianças de 1 a 14 anos, as neoplasias foram responsáveis por $8 \%$ dos óbitos, representando, assim, a quarta causa de morte (excluindo os óbitos por afecções mal definidas), sucedendo às causas externas, às doenças do aparelho respiratório e às doenças infecciosas (DATASUS, 1998).

Ao se compararem as taxas de mortalidade por câncer infantil observadas nas Américas, Ásia e Oceania (Tabela 4), constatou-se que alguns países da América Latina (Uruguai, Cuba, Argentina, Costa Rica), o Kuwait, a Nova Zelândia e Cingapura possuem as maiores taxas (Levi et al., 1995). Contudo, estas mostram-se ainda inferiores às taxas mais altas encontradas em países europeus (Levi et al., 1992).

Verifica-se que Porto Rico apresenta as menores taxas de mortalidade por neoplasias na infância para o sexo masculino $(30,3$ por um milhão de meninos), enquanto a Bulgária apresenta as maiores $(82,3)$; já no sexo feminino, destacam-se o Sri-Lanka (27,3 por um milhão de meninas) e Cingapura $(60,7)$.

Dos óbitos por neoplasias na infância, as leucemias representam a maior causa, sendo responsáveis por $39 \%$ das mortes na Europa (Martos \& Olsen, 1993) e por 50\% nas Américas, Oceania e Ásia (Levi et al., 1995). As maiores taxas são encontradas em Cingapura (30,7 e 25,8 por um milhão de meninos e de meninas, respectivamente), e as menores, em Porto Rico (11,4 por um milhão de meninos) e em Israel (8,6 por um milhão de meninas).

Embora se saiba que as taxas de mortalidade não retratam diretamente a atenção à saúde no que se refere ao câncer, a análise de tendências de mortalidade por câncer infantil pode
Tabela 4

Coeficientes padronizados* de mortalidade anual de câncer infantil, segundo área, período e sexo (por um milhão de habitantes $<15$ anos).

\begin{tabular}{|c|c|c|c|}
\hline Área & Período & Masculino & Feminino \\
\hline \multicolumn{4}{|l|}{ América ${ }^{a}$} \\
\hline Estados Unidos & $1985-89$ & 41,6 & 34,9 \\
\hline Canadá & $1985-89$ & 48,7 & 37,6 \\
\hline Uruguai & $1985-89$ & 73,6 & 51,2 \\
\hline Cuba & $1985-88$ & 66,4 & 59,2 \\
\hline Argentina & $1985-87$ & 69,9 & 58,1 \\
\hline Brasilc & $1978-96$ & 83,7 & 64,6 \\
\hline Costa Rica & $1985-88$ & 67,6 & 53,7 \\
\hline México & $1985-86$ & 54,7 & 43,7 \\
\hline Venezuela & $1985-87$ & 59,2 & 51,0 \\
\hline Porto Rico & $1985-88$ & 30,3 & 39,4 \\
\hline \multicolumn{4}{|l|}{ Europab } \\
\hline Bulgária & $1985-89$ & 82,3 & 58,4 \\
\hline Portugal & $1985-89$ & 74,6 & 59,7 \\
\hline Itália & $1985-89$ & 62,2 & 49,4 \\
\hline França & $1985-89$ & 55,6 & 44,4 \\
\hline Reino Unido (Escócia) & 1985-89 & 42,6 & 34,1 \\
\hline Áustria & $1985-89$ & 42,4 & 35,9 \\
\hline \multicolumn{4}{|l|}{ Ásiaa } \\
\hline Kuwait & $1985-87$ & 72,0 & 50,4 \\
\hline Cingapura & $1985-89$ & 65,3 & 60,7 \\
\hline Israel & $1985-89$ & 45,5 & 33,4 \\
\hline Japão & $1985-89$ & 49,7 & 40,8 \\
\hline Sri-Lanka & $1985-86$ & 33,6 & 27,3 \\
\hline \multicolumn{4}{|l|}{ Oceania } \\
\hline Austrália & $1985-88$ & 46,1 & 39,2 \\
\hline Nova Zelândia & $1985-89$ & 66,9 & 53,2 \\
\hline
\end{tabular}

*população padrão: mundial de Segi de 1960

Fonte: a Levi et al., 1995; b Levi et al., 1992; c Braga, 2000.

ser um indicador da eficácia de estratégias de intervenção contra o câncer. Na Tabela 3, resumem-se as tendências de mortalidade por câncer infantil em diferentes regiões do mundo.

Durante os últimos trinta anos, houve um declínio superior a $50 \%$ na mortalidade por câncer na infância, nos Estados Unidos, Canadá, Austrália e Nova Zelândia (La Vecchia et al., 1998; Levi et al., 1995); de 40\% na Europa Ocidental e de aproximadamente $20 \%$ na Europa Oriental (Kunze et al., 1997; Levi et al., 1992, 1995; Martos \& Olsen, 1993). No que tange à Europa, esse declínio ocorreu mais precoce e acentuadamente na Região Norte do que nas Regiões Sul e Leste, fazendo-se supor que a incorporação de novas estratégias terapêuticas para o câncer infantil possa ter-se dado de for- 
ma desigual em diversas regiões européias (Levi et al., 1992).

Evidências de declínio nos coeficientes de mortalidade também foram percebidas em Porto Rico, Israel e Japão. Em Bombaim, Índia, as taxas de mortalidade por neoplasias diminuíram acentuadamente (40\%) em duas décadas (1964-84) entre os menores de cinco anos (Krishnamurthy \& Dhar, 1991). Na Colômbia, Panamá e Venezuela, ao contrário, a mortalidade por câncer infantil mostrou-se estável, ao passo que, no México, observou-se aumento (Levi et al., 1995).

A tendência observada na mortalidade por neoplasias malignas em menores de 15 anos muitas vezes pode ser explicada pela tendência na mortalidade por leucemias. La Vecchia et al. (1998) observaram que, nas regiões economicamente desenvolvidas (América do Norte, Europa Ocidental, Japão e Oceania), as taxas de mortalidade por leucemias decresceram mais de $55 \%$ nas três últimas décadas.

Em estudo recente com a população dos Estados Unidos, Linet et al. (1999) atribuíram esse resultado principalmente à melhora na sobrevida das leucemias linfocíticas agudas.

$\mathrm{Na}$ Áustria, as taxas de mortalidade por tumores na infância decresceram entre 1980 e 1992, principalmente em decorrência da diminuição das taxas de mortalidade por leucemias, grupo este que compreendeu $34,5 \%$ de todas neoplasias malignas nessa faixa etária. Outros grupos de diagnóstico não apresentaram redução significativa de suas taxas (Kunze et al., 1997).

Na ex-União Soviética, a tendência de mortalidade por câncer infantil foi similar à observada na mortalidade por leucemias, com taxas estáveis entre 1965 e 1985 e decréscimo nos cinco anos seguintes (La Vecchia et al., 1994). Na Itália, por sua vez, entre o início da década de 60 e final da década de 80 , as taxas de mortalidade por todas as neoplasias malignas combinadas e somente por leucemias apresentaram declínio superior a 50\%, porém, entre 1990 e 1992, não houve evidência de declínio e, sim, uma possível reversão nessa tendência (La Vecchia et al., 1995, 1996).

Em Goiânia, entre 1978 e 1996, as taxas de mortalidade por neoplasias infantis apresentaram decréscimo com significância estatística para as crianças com idade igual ou superior a cinco anos, o mesmo tendo ocorrido em relação à mortalidade por leucemias entre os menores de 15 anos no período de 1979 a 1995 (Braga, 2000). Todavia, na análise conjunta para todas as faixas etárias e todos os tumores da infância, a tendência foi de estabilidade.
Coleman et al. (1993) acreditam que, não obstante o declínio na mortalidade por câncer ser praticamente universal em menores de 15 anos, tal redução não se faz sentir de modo uniforme em todas as populações e grupos étnicos. Infelizmente, os avanços na terapêutica do câncer não se encontram disponíveis em todos os hospitais, dificultando, assim, o acesso de muitas crianças aos novos tratamentos (Robertson et al., 1994). Além das diferenças regionais anteriormente citadas, cabe destacar a existência de discrepâncias no prognóstico do câncer infantil em relação a variáveis sócio-demográficas. Pui et al. (1995), comparando os desfechos alcançados no tratamento do câncer de crianças no hospital Saint Jude entre 1962 e 1992, observaram menor probabilidade de sobrevida para crianças negras, particularmente no início do período analisado.

\section{Sobrevida}

O declínio observado, em diversos países, nos coeficientes de mortalidade por neoplasias em menores de 15 anos parece dever-se, em grande parte, ao aumento da probabilidade de sobrevida para a maioria dos casos com tumores infantis, ou seja, ao acréscimo na porcentagem de crianças vivas com neoplasmas após um determinado período de tempo, em decorrência de diagnósticos mais precoces e maior sucesso nas intervenções terapêuticas (radioterapia, quimioterapia, cirurgia, transplante de medula óssea) (Latorre \& Franco, 1996). Os recentes avanços no uso dos medicamentos quimioterápicos e o emprego de esquemas combinados de drogas permitiram elevar a sobrevida de crianças com neoplasias, particularmente as hematológicas (Latorre, 2000; Ries et al., 1999a).

Existe uma diferença substancial entre as probabilidades de sobrevida observadas em pacientes participantes de ensaios clínicos terapêuticos em centros oncológicos especializados e as assinaladas em registros de base populacional. Embora as primeiras sejam geralmente maiores, admite-se que as probabilidades de sobrevida de base populacional reflitam mais acuradamente as ações de saúde rotineiramente adotadas numa determinada população, tais como: medidas de prevenção, métodos de rastreamento visando ao diagnóstico mais precoce, eficácia do tratamento e até mesmo o seguimento pós-tratamento (Kramárová et al., 1996).

As probabilidades de sobrevida acumuladas após cinco anos em diversos países podem ser observadas na Tabela 5. As taxas obtidas nos re- 
Probabilidades de sobrevida após cinco anos (em \%) para o câncer infantil no mundo.

\begin{tabular}{|c|c|c|c|c|c|c|c|}
\hline Neoplasia & $\begin{array}{l}\text { Goiâniaa } \\
\text { 1989-94 }\end{array}$ & $\begin{array}{l}\text { Namíbiab } \\
1983-88\end{array}$ & $\begin{array}{c}\text { Eslováquiac } \\
\text { 1983-87 }\end{array}$ & $\begin{array}{l}\text { Alemanhad } \\
1987-92\end{array}$ & $\begin{array}{l}\text { Itáliae } \\
1986-89\end{array}$ & $\begin{array}{c}\text { Inglaterraf } \\
\text { (sudoeste) } \\
1983-87\end{array}$ & $\begin{array}{c}\text { Estados Unidos } \\
1983-90\end{array}$ \\
\hline $\begin{array}{l}\text { Todas as neoplasias } \\
\text { combinadas malignas }\end{array}$ & 48 & 37 & 46 & 70 & 69 & 64 & 70 \\
\hline Leucemias & 27 & 39 & 39 & 71 & 67 & 68 & - \\
\hline Linfomas & 67 & - & 55 & 86 & - & 76 & - \\
\hline Tumores do SNC & 39 & 25 & 40 & 55 & 65 & 57 & 62 \\
\hline
\end{tabular}

Fontes: a Braga (2000); b Wessels \& Hesseling (1996); c Kramárová et al. (1996); d Kaatsch et al. (1995);

e Magnani et al. (1997); f Foreman et al. (1996); g Ries (1999b) (sobrevida relativa); SNC = sistema nervoso central.

gistros da Eslováquia e Namíbia foram, em geral, inferiores às dos países desenvolvidos.

Nos Estados Unidos, por exemplo, notou-se que a probabilidade de uma criança sobreviver cinco anos após o diagnóstico de neoplasia passou de $28 \%$ (em 1960) para aproximadamente 70\% (no final da década de 80) (Franco, 1997; Greenlee et al., 2001; Novakovic, 1994; Ries, 1999b). Na Itália, entre 1978 e 1989, essa mesma taxa foi de $61 \%$ (Magnani et al., 1997), e, na Alemanha, de 70\% entre 1987 e 1992 (Kaatsch et al., 1995).

Incrementos nas probabilidades de sobrevida de todas as neoplasias combinadas também foram observados na Austrália. Em Queensland, passaram de 31\% (1956-66) para 52\% (1977-80), e, em Victoria, de 49\% (1970-79) para 67\% (1980-89) (Giles et al., 1995; McWhirter \& Siskind, 1984). Da mesma forma, Braga (2000) verificou que a sobrevida acumulada após cinco anos, em Goiânia, aumentou de $35,1 \%$ (1989-90) para 73,4\% (1993-94).

Stiller \& Bunch (1990) acreditam que isso se deve aos avanços no tratamento de vários tipos de câncer em crianças, principalmente no que diz respeito às LLA. Há quarenta anos, apenas $10 \%$ das crianças sobreviviam cinco anos após o diagnóstico. Hoje, ao contrário, a maioria das crianças pode ser curada num curto espaço de tempo. Nos Estados Unidos, por exemplo, a sobrevida após cinco anos de crianças com LLA aumentou de 4\% (1960-1963) para 69\% (19831987) (Novakovic, 1994), e, mais recentemente, para 82\% (1989-1996) (Greenlee et al., 2001); na Eslováquia, de 7,5\% (1968-72) para 50\% (198387) (Kramárová et al., 1996), e, em Queensland (Austrália), de 2,2\% (1956-66) para 48\% (197780) (McWhirter \& Siskind, 1984).

Analogamente, em quase todos os grupos de diagnóstico, tem-se apresentado aumento nas probabilidades de sobrevida (Bleyer, 1990).
No entanto, acentuada variabilidade pode ocorrer entre as probabilidades de sobrevida dos pacientes diagnosticados com diferentes tumores, considerando a história natural da doença conforme o órgão afetado e respostas variadas à terapêutica antineoplásica (Franco, 1994). As neoplasias pediátricas mostram-se habitualmente fatais quando não tratadas adequadamente ou em tempo hábil.

Apesar dos grandes avanços tecnológicos e científicos na abordagem do câncer infantil, em países em desenvolvimento a probabilidade de sobrevida a essa doença é ainda muito baixa: 37,5\% em Bangalore, Índia (Nandakumar et al., 1996); 37\% na Namíbia, África (Sriamporn et al., 1996) e 48\% no Município de Goiânia, Brasil (Braga, 2000).

Apesar de, em muitos países, ter-se verificado aumento na incidência das neoplasias em menores de 15 anos e de o prognóstico para vários tumores infantis ainda estar aquém do desejado, sabe-se que a sobrevida das crianças com câncer é melhor hoje do que outrora (Schmidt, 1998). Particularmente, para alguns tipos histológicos, o aumento da sobrevida fezse de forma mais marcante. Coleman et al. (1993) acreditam que a sobrevida de cinco anos à LLA ter passado de $37 \%$ para $75 \%$ foi um dos maiores contribuintes para o declínio da mortalidade por câncer em crianças na Inglaterra no período entre 1971 e 1985.

Sabe-se, no entanto, que diversos fatores podem interferir nas probabilidades de sobrevida ao câncer infantil. Na Índia, por exemplo, as crianças chegam aos centros especializados em estágios avançados da doença, reduzindo, portanto, as chances de sucesso do tratamento (Nandakumar et al., 1996). De forma análoga, as baixas probabilidades de sobrevida observadas em crianças da Namíbia têm sido atribuídas a possível retardo no estabelecimento do 
diagnóstico (Wessels \& Hesseling, 1996). A demora na procura de cuidados médicos, por sua vez, pode dever-se a diversas variáveis de natureza sócio-econômica, tais como menor acesso aos serviços de saúde, barreiras culturais que dificultam a percepção da possibilidade de cura do câncer e, até mesmo, barreiras lingüísticas, como ocorre em minorias raciais vivendo em países desenvolvidos (Hampton, 1998).

Outro fator a ser considerado diz respeito à aderência dos pacientes ao tratamento. Particularmente em relação ao câncer infantil, ela pode estar reduzida, em virtude da toxicidade dos esquemas terapêuticos. Nesse sentido, cabe destacar a importância da inclusão de pacientes em protocolos de ensaios clínicos. Em tais circunstâncias, os pacientes são acompanhados com mais rigor, garantindo-se maior aderência (Kramárová et al., 1996). Bleyer (1997) enfatizou que, nos Estados Unidos, o desenvolvimento de um programa nacional de ensaios clínicos multicêntricos alterou substancialmente o prognóstico do câncer infantil, revertendo taxas de mortalidade de $80 \%$ para sobrevida de $80 \%$ nas últimas quatro décadas.

\section{Agradecimentos}

À Fundação de Amparo à Pesquisa do Estado de São Paulo (Processo no 98/04608-1) e ao Conselho Nacional de Pesquisa e Desenvolvimento Tecnológico (Processo no 300318/97-9), pelas bolsas de estudo.

\section{Conclusões}

Em resumo, procurou-se, no presente artigo, revisar os estudos epidemiológicos mais recentes de incidência de tumores na infância, mortalidade e sobrevida, comparando os resultados encontrados em Goiânia com aqueles obtidos em outros países. Em geral, a incidência das neoplasias infantis encontra-se estável, ao passo que a mortalidade apresenta tendência de declínio, principalmente nos países desenvolvidos, como provável decorrência do aumento das probabilidades de sobrevida acumuladas, observado nas últimas décadas.

A avaliação criteriosa desses dados permite identificar grupos populacionais sob maior risco ou com prognóstico mais desfavorável, servindo às autoridades sanitárias e aos pesquisadores na área de câncer como instrumento relevante na definição das ações de saúde necessárias para o adequado controle e prevenção da doença.

\section{Referências}

BLACK, W. C., 1998. Increasing incidence of childhood primary malignant brain tumors-enigma or no-brainer? Journal of the National Cancer Institute, 90:1249-1251.

BLEYER, W. A., 1990. The impact of childhood cancer on the US and the world. CA-A Cancer Journal for Clinicians, 40:355-367.

BLEYER, W. A., 1997. The U.S. pediatric cancer clinical trials programmes: International implications and the way forward. European Journal of Cancer, 33:1439-1447.

BRAGA, P. E. B., 2000. Câncer na Infância: Tendências e Análise de Sobrevida em Goiânia (1989-1996). Dissertação de Mestrado, São Paulo: Faculdade de Saúde Pública, Universidade de São Paulo. 
BRESLOW, N. E. \& LANGHOLZ, B., 1983. Childhood cancer incidence: Geographical and temporal variations. International Journal of Cancer, 32: 703-716.

BUNIN, G. R.; FEUER, E. J.; WITMAN, P. A. \& MEADOWS, A. T., 1996. Increasing incidence of childhood cancer: Report of 20 years experience from the Greater Delaware Valley Pediatric Tumor Registry. Paediatric and Perinatal Epidemiology, 10: 319-338.

COLEMAN, M. P.; ESTEVE, J.; DAMIECKI, P.; ARSLAN, A. \& RENARD, H., 1993. Trends in Cancer Incidence and Mortality. IARC Scientific Publications 121. Lyon: International Agency for Research on Cancer/World Health Organization.

DATASUS (Departamento de Informática do Sistema Único de Saúde), 1998. Sistema de Informações sobre Mortalidade; 1979 - 1997: Dados de Declaração de Óbito. CD-ROM. Brasília: Ministério da Saúde.

DE NULLY-BROWN, P.; HERTZ, H.; OLSEN, J.H.; YSSING, M.; SCHEIBEL, E. \& JENSEN, O. M., 1989. Incidence of childhood cancer in Denmark 1943-1984. International Journal of Epidemiology; 18:546-555.

DESMEULES, M.; MIKKELSEN, T. \& MAO, Y., 1992. Increasing incidence of primary malignant brain tumors: Influence of diagnostic methods. Journal of the National Cancer Institute, 84:442-445

FOREMAN, N. K.; THORNE, R. N. \& MOTT, M. G., 1996. Variation in survival of children with cancer within a region of the United Kingdom. Cancer, 77:785-790.

FRANCO, E., 1994. Cancer epidemiology: Substance and methods. Ciência e Cultura, 46:46-62.

FRANCO, E., 1997. Epidemiology in the study of cancer. In: Encyclopedia of Cancer (J. Bertino, ed.), pp. 621-641, New York: Academic Press.

FUNDAÇÃO ANTÔNIO PRUDENTE, 1997. Tumores Pediátricos. Registro Hospitalar de Câncer. 26 Março 2001 <http://www.hcanc.org.br>.

GIBBONS, L.; MAO, Y.; LEVY, I. G. \& MILLER, A. B., 1994. The Canadian Childhood Cancer Control Program. Canadian Medical Association Journal, 151:1704-1709.

GILES, G.; WATERS, K.; THURSFIELD, V. \& FARRUGIA, H., 1995. Childhood cancer in Victoria, Australia, 1970-1989. International Journal of Cancer, 63: 794-797.

GREENBERG, R. S. \& SHUSTER, J. L., 1985. Epidemiology of cancer in children. Epidemiologic Reviews, 7:22-48.

GREENLEE, R. T.; HILL-HARMON, M. B.; MURRAY, T. \& THUN, M., 2001. Cancer statistics, 2001. CA Cancer Journal of Clinics, 51:15-36.

GURNEY, J. G.; DAVIS, S.; SEVERSON, R. K.; FANG, J. Y.; ROSS, J. A. \& ROBISON, L. L., 1996. Trends in cancer incidence among children in the U.S. Cancer, 78:532-541.

GURNEY, J. G.; ROSS, J. A.; WALL, D. A.; BLEYER, W. A.; SEVERSON, R. K. \& ROBISON, L. L., 1997. Infant cancer in the U.S.: Histology-specific incidence and trends, 1973 to 1992. Journal of Pediatric Hematology/Oncology, 19:428-432.

HAMPTON, J. W., 1998. The disproportionately lower cancer survival rate with increased incidence and mortality in minorities and underserved americans. Cancer, 83:1687-1690.

JENSEN, O. M. \& STORM, H. H., 1991. Purposes and uses of cancer registration. In: Cancer Registration Principles and Methods (O. M. Jensen, D. M. Parkin, R. Maclennan, C. S. Muir \& R. G. Skeet, ed.), pp. 7-21, IARC Scientific Publications 95. Lyon: International Agency for Research on Cancer.

KAATSCH, P.; HAAF, G. \& MICHAELIS, J., 1995. Childhood malignancies in Germany-methods and results of a nationwide registry. European Journal of Cancer, 31A:993-999.

KRAMÁROVÁ, E.; PLESKO, I.; BLACK, R. J. \& OBSITNÍKOVÁ, A., 1996. Improving survival for childhood cancer in Slovakia. International Journal of Cancer, 65:594-600.

KRISHNAMURTHY, S. \& DHAR, M., 1991. Cancer and other causes of childhood mortality in Bombay, India. Cancer, 68:1848-1853.

KUNZE, U.; WALDHOER, T. \& HAIDINGER, G., 1997. Childhood cancer mortality in Austria, 1980-1992. European Journal of Epidemiology, 13:41-44.

LA VECCHIA, C.; LEVI, F. \& LUCCHINI, F., 1994. Trends in childhood cancer mortality in the exUSSR, 1965-1990. International Journal of Cancer, 57:765-766.

LA VECCHIA, C.; LEVI, F.; LUCCHINI, F.; LAGIOU, P.; TRICHOPOULOS, D. \& NEGRI, E., 1998. Trends in childhood cancer mortality as indicators of the quality of medical care in the developed world. Cancer, 83:2223-2227.

LA VECCHIA, C.; NEGRI, E. \& DECARLI, A., 1995. Childhood cancer mortality in Italy. Revue d'Epidémiologie et de Santé Publique, 43:383-384.

LA VECCHIA, C.; NEGRI, E. \& DECARLI, A., 1996. Childhood cancer mortality in Italy. Lancet, 347 : 1633-1634.

LATORRE, M. R. D. O., 1992. Mortalidade como Preditor da Morbidade. Dissertação de Mestrado, São Paulo: Faculdade de Saúde Pública, Universidade de São Paulo.

LATORRE, M. R. D. O., 2000. Epidemiologia dos tumores na infância. In: Pediatria Oncológica (B. Camargo \& L. F. Lopes, org.), pp. 7-27, São Paulo: Lemar.

LATORRE, M. R. D. O. \& FRANCO, E. L., 1996. Epidemiologia dos tumores na infância. Acta Oncológica Brasileira, 16:201-219.

LEVI, F.; LA VECCHIA, C.; LUCCHINI, F.; NEGRI, E. \& BOYLE, P., 1992. Patterns of childhood cancer incidence and mortality in Europe. European Journal of Cancer, 28A:2028-2049.

LEVI, F.; LA VECCHIA, C.; LUCCHINI, F; NEGRI, E. \& BOYLE, P., 1995. Patterns of childhood cancer mortality: America, Asia and Oceania. European Journal of Cancer, 31A:771-782.

LINET, M. S.; RIES, L. A. G.; SMITH, M. A.; TARONE, R. E. \& DEVESA, S. S., 1999. Cancer surveillance series: Recent trends in childhood cancer incidence and mortality in the United States. Journal of the National Cancer Institute, 91:1051-1058.

LITTLE, J., 1999. Epidemiology of Childhood Cancer. IARC Scientific Publications 149. Lyon: International Agency for Research on Cancer.

MAGNANI, C. \& PASTORE, G., 1997. Survival of childhood cancer patients in Italy, 1978-1989. Tumori, 83:426-429. 
MALKIN, D., 1997. Cancers of childhood. In: Cancer: Principles and Practice of Oncolology (V. T. De Vita, S. Hellman Jr., \& S. A. Rosenberg, ed.), pp. 2083-2091, 5th Ed. New York: Lippincott Raven.

MARTOS, M. C. \& OLSEN, J. H., 1993. Childhood cancer mortality in the European Community, 19501989. European Journal of Cancer; 29A:1783-1789.

McWHIRTER, W. R.; DOBSON, C. \& RING, I., 1996. Childhood cancer incidence in Australia, 19821991. Internationalt Journal of Cancer; 65:34-38.

McWHIRTER, W. R. \& SISKIND, V., 1984. Childhood cancer survival trends in Queensland 1956-80. British Journal of Cancer, 49:513-519.

MILLER, R. W.; YOUNG, J. L. \& NOVAKOVIC, B., 1995. Childhood cancer. Cancer, 75(Sup. 1):395-405.

MOSSO, M. L.; COLOMBO, R.; GIORDANO, L.; PASTORE, G.; TERRACINI, B. \& MAGNANI, C., 1992. Childhood cancer registry of the Province of Torino, Italy. Cancer, 69:1300-1306.

NANDAKUMAR, A.; ANANTHA, N.; APPAJI, L.; SWAMY, K.; MUKHERJEE, G.; VENUGOPAL, T.; REDDY, S. \& DHAR, M., 1996. Descriptive epidemiology of childhood cancers in Bangalore, India. Cancer Causes Control, 7:405-410.

NOVAKOVIC, B., 1994. U.S. childhood cancer survival, 1973-1987. Medical and Pediatric Oncology, 23:480-486.

PARKIN, D. M.; KRÁMAROVÁ, E.; DRAPER, G. J.; MASUYER, E.; MICHAELIS, J.; NEGLIA, J.; QURESHI S. \& STILLER C. A. (ed.), 1998. International Incidence of Childhood Cancer. v. 2, IARC Scientific Publications 144. Lyon: International Agency for Research on Cancer/World Health Organization.

PARKIN, D. M.; PISANI, P. \& FELAY, J., 1999. Global Cancer Statistics. 26 March $2001<$ http://www.cajournal.org/>.

PARKIN, D. M.; STILLER, C. A.; DRAPER, G. J. \& BIEBER, C. A., 1988a. The international incidence of childhood cancer. International Journal of Cancer, 42:511-520.

PARKIN, D. M.; STILLER, C. A.; DRAPER, G. J.; BIEBER, C. A.; TERRACINI, B. \& YOUNG, J. L., 1988b. International Incidence of Childhood Cancer. IARC Scientific Publications 87. Lyon: International Agency for Research on Cancer.

PETRILLI, A. S.; CARNEIRO Jr., J. L.; CYPRIANO, M.; ANGEL, A. \& TOLEDO, S., 1997. Diferenças clínicas, epidemiológicas e biológicas entre o câncer na criança e no adulto. Revista Brasileira de Cancerologia, 43:191-203.

POLLAN, M.; LOPEZ-ABENTE, G.; ARDANAZ, E.; MOREO, P.; MORENO, C.; VERGARA, A. \& ARAGONES, N., 1997. Childhood cancer incidence in Zaragoza and Navarre (Spain): 1973-1987. European Journal of Cancer, 33:616-623.

PUI, C. H.; BOYETT, J. M.; HANCOCK, M. L.; PRATT, C. B.; MEYER, W. H. \& CRIST W. M., 1995. Outcome of treatment for childhood cancer in black as compared with white children. The St. Jude Children's Research Hospital experience, 1962 through 1992. JAMA, 273:633-637.
RIES, L. A. G., 1996. Cancer Among Children Under Age 15. 8 November 1999 <http://www.nci.nih. gov/>.

RIES, L. A. G.; SMITH, M. A.; GURNEY, J. G.; TAMRA, T.; YOUNG, J. L. \& BUNIN, G. R., 1999a. Cancer Incidence and Survival among Children and Adolescents: United States SEER Program 1975-1995. Bethesda: National Cancer Institute.

ROBERTSON, C. M.; HAWKINS, M. M. \& KINGSTON, J. E., 1994. Late deaths and survival after childhood cancer: Implications for cure. BMJ, 309:162166.

SCHMIDT, C. W., 1998. Childhood cancer: A growing problem. Environmental Health Perspectives, 106:A18-23.

SHARP, L.; COTTON, A. \& LITTLE, J., 1999. Descriptive epidemiology. In: Epidemiology of Childhood Cancer (J. Little, ed.), pp.10-66, IARC Scientific Publications 149. Lyon: International Agency for Research on Cancer/World Health Organization.

SMITH, M. A.; FREIDLIN, B.; RIES, L. A. G. \& SIMON, R., 1998. Trends in reported incidence of primary malignant brain tumors in children in the United States. Journal of the National Cancer Institute, 90:1269-1277.

SRIAMPORN, S.; VATANASAPT, B.; MARTIN, N.; SRIPLUNG, H.; CHINDAVIJAK, K.; SONTIPONG, S.; PARKIN, D. M. \& FERLAY, J., 1996. Incidence of childhood cancer in Thailand 1988-1991. Paediatric and Perinatal Epidemiology, 10:73-85.

STILLER, C. A. \& BUNCH, K. J., 1990. Trends in survival for childhood cancer in Britain diagnosed 1971-85. British Journal of Cancer, 62:806-815.

WABINGA, H. R.; OWOR, R. \& NAMBOOZE, S., 1998. Kampala cancer registry, 1992-1995. International Incidence of Childhood Cancer (D. M. Parkin, E. Kramárová, G. J. Draper, E. Masuyer, J. Michaelis, J. Neglia, S. Qureshi \& C. A. Stiller, ed.), p. 53, v. 2, IARC Scientific Publications 144. Lyon: International Agency for Research on Cancer/World Health Organization.

WESSELS, G. \& HESSELING, P. B., 1996. Outcome of children treated for cancer in the Republic of Namibia. Medical and Pediatric Oncology, 27: 160-164.

WESSELS, G.; HESSELING, P. B. \& KUIT, S. B., 1998. The Namibia children's tumour registry, 19831992. In: International Incidence of Childhood Cancer ((D. M. Parkin, E. Kramárová, G. J. Draper, E. Masuyer, J. Michaelis, J. Neglia, S. Qureshi \& C. A. Stiller, ed.), p. 39, v. 2, IARC Scientific Publications 144. Lyon: International Agency for Research on Cancer/World Health Organization.

Recebido em 8 de agosto de 2000

Versão final reapresentada em 16 de abril de 2001

Aprovado em 7 de junho de 2001 